\title{
A Cluster Head Routing Protocol for Improving Network Lifetime in Wireless Sensor Networks
}

\author{
Dr. Gaurav Sharma \\ Asst. Professor, CVR College of Engineering/ECE Department, Hyderabad, India \\ Email: ergaurav209@yahoo.co.in
}

\begin{abstract}
The energy efficiency in wireless sensor networks (WSNs) is a fundamental challenge. Cluster based routing is an energy saving method in this type of networks to reduce energy consumption in WSNs. A new Energy Efficient Cluster Head Routing protocol for heterogeneous WSN, which is called CHRP is proposed and evaluated which works on sleep-awake policy that helps in prolonging lifetime of the network. In CHRP, the cluster head is elected if its residual energy is more than system average energy of the network. Node pairing mechanism is also employed in CHRP. The nodes with high initial and residual energy will have more chances to become cluster head. Finally, the simulation results show that CHRP enhances lifetime of heterogeneous sensor network as compared to other protocols i.e., Distributed Energy Efficient Clustering (DEEC) and Threshold Sensitive Stable Electron Protocol (TSEP).
\end{abstract}

Index Terms: Clustering, Routing Protocol, Energy Efficient Algorithms, Wireless Sensor Networks.

\section{INTRODUCTION}

Wireless Sensor Networks (WSNs) have gained worldwide attention in recent years, particularly with recent advances in microelectromechanical systems (MEMS) technology, wireless communications and digital electronics which have enabled the development of low-cost, lowpower and multifunctional sensor nodes. A WSN consists of many small and low-cost sensor nodes capable of detecting physical phenomena such as temperature, light, heat, sound, etc. $[1,2]$. WSNs have many applications including military, monitoring, home applications, traffic control, etc. Since the sensor nodes may be applied in dangerous and inaccessible environments, replacing or recharging their power supplies is not possible and economic. Therefore, optimizing energy consumption for prolonging the network lifetime is a critical issue in WSNs. In order to prolong the lifetime of sensor network, energy efficient routing protocols are designed based on clustering techniques.

Clustering has been proved an effective routing method for reducing the energy dissipation of nodes, balancing the energy consumption among nodes and prolonging the network lifetime $[3,4]$. In clustering methods, nodes are divided into groups called clusters. Each cluster has a leader node named cluster head $(\mathrm{CH})$ and the rest of the nodes are called member nodes. Member nodes transmit their data to the corresponding $\mathrm{CH}$ and then the $\mathrm{CH}$, after collecting the received data, aggregates their data together with its own data and transmits them to the base station (BS).

In general, the clustering process consists of two phases: the setup phase and the steady state phase. The setup phase includes the selection of $\mathrm{CHs}$ and forming the clusters. In the steady state phase, member nodes transmit their data to their corresponding $\mathrm{CH}$, and $\mathrm{CH}$ sends the aggregated data to BS. Since most of the cluster-based routing protocols do not consider the consumed energy due to the clusters formation in the setup phase, the energy dissipation is not properly optimized.

In this paper, the performance of CHRP algorithm in saving energy for heterogeneous WSNs is studied. In the sensor network considered in this paper, each node transmits data either directly to base station or via cluster head which is elected by certain clustering algorithm, fuses the data collected from their cluster members and sends it to the "sink node".

Here in this protocol, all the sensor nodes with different amounts of energy which leads to heterogeneous sensor network is considered [5]. The addition of new nodes in the networks prolongs the lifetime of the network. Currently in most of the protocols, such as DEEC [1], TSEP [6], heterogeneous network is assumed. In TSEP [6] three levels of heterogeneous sensor network is taken which is composed of normal nodes, intermediate nodes and advanced nodes. The advanced nodes are equipped with more energy nodes than normal nodes. Also, cluster heads are selected depending on probability of each type of node. However, it is not fit for multilevel heterogeneous WSN that includes more than two types of nodes.

In DEEC [1], each node spends its energy uniformly by rotating the role of cluster head among all nodes. Basically, $\mathrm{CHs}$ are elected based on the ratio between residual energy of each node and average energy of the network. Thus, DEEC can improve the stability period and hence prolong the network lifetime by heterogeneous clustering approach.

In this paper, a new energy efficient cluster head routing algorithm for heterogeneous WSN composing three different types of nodes i.e. normal nodes, super nodes and advanced nodes is proposed and evaluated. In CHRP, basically sleepaware policy is used in which some nodes put to sleep to conserve energy and hence prolongs the lifetime of the network. Utilizing the concept of node pairing among sensor nodes and threshold energies, energy utilization of the network is optimized. The remainder of the paper is organized as follows. Section II describes the proposed algorithm. Section III shows the performance of CHRP by simulation and compares it with DEEC and TSEP. Finally, section IV gives concluding remarks.

\section{Proposed WORK}

This section describes a new Algorithm CHRP (Cluster Head Routing Protocol) which has two main characteristics: 


\section{Direct Communication}

\section{Transmission via Cluster Head}

It is reactive routing protocol, as transmission consumes more energy than sensing and it is done only when a precise threshold is reached $[8,9]$.

To describe whole protocol clearly particularly discussion about energy model and how optimum number of clusters can be computed is done. For three levels of heterogeneity, nodes with different energy levels are:

1) Normal Nodes

2) Advance Nodes

\section{3) Super Nodes}

Advance nodes having energy greater than normal nodes, super nodes with the highest energy. Super nodes can be chosen by using $b$, where $b$ is proportion of super nodes and using the relation that energy of normal nodes is $\mu$ times more than that of normal nodes. Energy for normal nodes is $E o$, for advance nodes it is $E_{A D V}=E O(1+\alpha)$ and energy for super nodes can be computed as $E_{S U P}=E o(1+\mu)$, where $\mu=$ $\alpha / 2$.

So total energy of normal nodes, advance nodes and for super nodes will be, n.Eo. $(1-m-b)$, n.m.Eo. $(1+\alpha)$, and n.b.Eo. $(1+\mu)$ respectively.

Therefore, the total Energy of all the nodes will be, $n E o .(1-m-b)+n \cdot m \cdot E o \cdot(1+\alpha)+n \cdot b \cdot E o .(1+\mu)=n \cdot E o(1$ $+m \alpha+b \mu)$.

where $n$ is number of nodes, $\mathrm{m}$ is proportion of advanced nodes to the total number of nodes $n$ with energy more than rest of nodes and $b$ is proportion of super nodes.

Starting of a round, BS broadcasts HELLO packets among the sensors periodically. If the RSSI of the received signal is greater than clustering threshold then no need to form clusters [10, 11, 12]. Based on stronger RSSI (Receiving Signal Strength Indicator) nodes closer to the BS are selected to send their data directly to BS. This region is called direct communication region. Remaining nodes follow dynamic clustering technique.

In WSN, sensors are deployed with high density due to limited resources and vulnerable nature of individual sensor. As a result the same area is covered by many sensor nodes. This causes a lot of redundancy because multiple sensor nodes consume energy to sense the same area and also to trans-receive the identical data. For this reason, redundant information will increase at the BS and also there will be wastage of transmission energy among the nodes. The solution to avoid this redundancy is to turn off the redundant nodes, as turning off some nodes does not affect the overall system as long as there are enough working nodes to provide the service. Turned-off sensor nodes save a significant amount of energy and this addresses one of the important constraints of WSN i.e. limited energy [13]. Therefore, if sensor nodes are scheduled to perform alternately, more energy can be saved and system lifetime can be prolonged effectively. So, in the proposed technique, at any moment, only some of the members perform i.e. they become active nodes and rest of the nodes remain in sleep mode i.e. they are passive nodes. The decision of choosing i.e. which node will be active and which will be passive is taken on the basis of RSSI. The proposed approach is explained using these three phases:

\section{A. Node Pairing}

Before performing clustering, a node has to select its nearest node. A node sends a request message to find nearest neighbor. The 1-hop neighbor nodes which are closer to that node send a reply with their distances from that particular node and they are included in eligible neighbor list. Then the node in the Eligible Neighbor List which has maximum RSSI is selected as next node $[14,15]$. The two nodes are added as coupled and then Node Paired ID message is broadcast in the network.

After performing the node pairing mechanism as shown in Fig.1, each node checks its remaining energy with its paired neighbor. In a pair, a node switches into Active mode if its residual energy is greater than its paired node. Thus, node having more residual energy in a pair will participate in clustering technique and the other one will remain in sleep mode for that round. During a sleeping period, the node ceases to perform any communication with the environment [14, 16, 17].

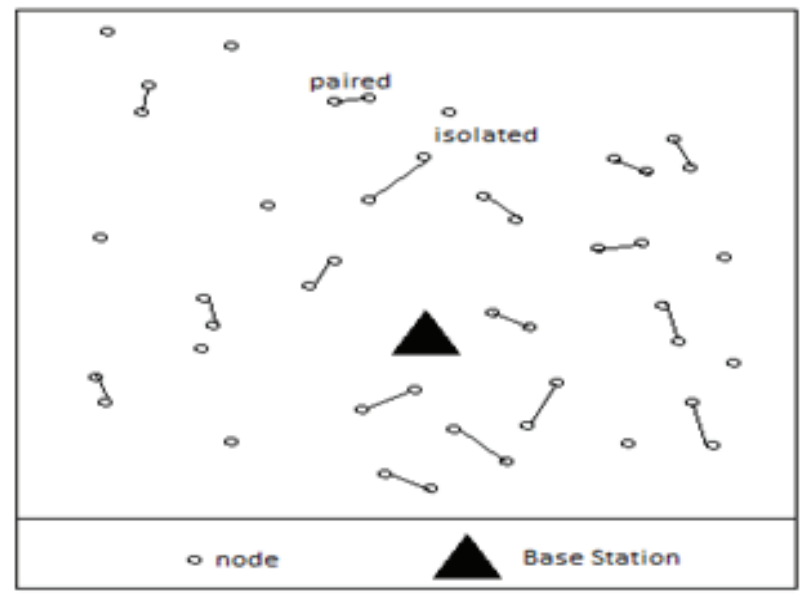

Figure 1. Node Pairing

Thus, power consumption is assumed to be minimal, whereas when a sensor is awake, it consumes regular amount of energy. In next communication period, nodes in Active-mode switch into Sleep-mode and Sleep-mode nodes switch into Active mode if and only if Sleep-mode node's residual energy is above Active-mode node's energy level. In this way, it is able to minimize energy consumption as nodes in Sleep-modes save their energy by not communicating with the CHs. Nodes in Sleep-mode also save their energy by avoiding idle listening and overhearing $[10,18,19]$. If coupled partner of a node is dead, then it will become active for rest of the round. Isolated nodes remain in Active-mode for every round till their energy resources depleted.

\section{B. Cluster Heads Selection}

At the starting of cluster head selection phase BS receives the status of the current energy level from all sensor nodes 
in the network. Then selection of powerful nodes is done based on the received energy values. The BS computes the average energy level of the active nodes as follows:

$$
E_{\text {avg }}=\sum \frac{E_{r s s i}}{m}
$$

where $m$ is the total number of active nodes; $E_{\text {resi }}$ is nodes residual energy.

So, CHRP protocol uses Eavg to be the main parameter for selecting $\mathrm{CHs}$ and handle well the heterogeneous energy capacities among the sensor nodes considering system average energy in each round. After BS broadcasts average energy of the network, nodes having remaining energy greater than or equal to the system average energy include themselves in the set of eligible cluster heads. If a node finds its Eresi $\geq$ Eavg then it sends a request message to find eligible neighbors $[7,12]$. The 1-hop neighbor nodes which are closer to that node send a reply with their Energy Consumption Rate Eecr in previous round. The node which has minimum energy consumption rate in previous round and with Eresi $\geq$ Eavg is selected as $\mathrm{CH}$ node, where energy consumption rate is as follows:

$$
E_{e c r}=\frac{E_{o}-E_{r e s i}}{m-1}
$$

The optimal probability of nodes, which are divided on the basis of energy, to be chosen as a $\mathrm{CH}$ can be calculated by using following formulas:

$$
\begin{aligned}
p_{\text {nrm }} & =\frac{p_{\text {opt }}}{1+m \cdot \alpha+b \cdot \mu} \\
p_{\text {adv }} & =\frac{p_{\text {opt }}(1+\alpha)}{1+m \cdot \alpha+b \cdot \mu} \\
p_{\text {sup }} & =\frac{p_{\text {opt }}(1+\mu)}{1+m \cdot \alpha+b \cdot \mu}
\end{aligned}
$$

Now to ensure that $\mathrm{CH}$ selection is done in the same way as it is assumed, another parameter is taken into consideration, which is threshold level. Each node generates a number randomly inclusive of 0 and 1 , if generated value is less than threshold then this node becomes $\mathrm{CH}[4,7]$. For all these type of nodes different formulas for the calculation of threshold depending on their probabilities are given below:

$$
\begin{aligned}
& T_{n r m}=\left\{\begin{array}{c}
\frac{p_{n r m}}{1-p_{n r m}\left[r \cdot \bmod \frac{1}{p_{n r m}}\right]}, \quad \text { if } n_{n r m} \in G^{\prime} \\
0, \quad \text { otherwise }
\end{array}\right. \\
& T_{a d v}=\left\{\begin{array}{c}
\frac{p_{a d v}}{1-p_{a d v}\left[r \cdot \bmod \frac{1}{p_{a d v}}\right]}, \quad \text { if } n_{a d v} \in G^{\prime \prime} \\
0, \quad \text { otherwise }
\end{array}\right. \\
& T_{\text {sup }}=\left\{\begin{array}{c}
\frac{p_{\text {sup }}}{1-p_{\text {sup }}\left[r \cdot \bmod \frac{1}{p_{\text {sup }}}\right]}, \quad \text { if } n_{\text {adv }} \in G^{\prime \prime \prime} \\
0, \quad \text { otherwise }
\end{array}\right.
\end{aligned}
$$

where $r$ is number of rounds.
$\mathrm{G}^{\prime}, \mathrm{G}^{\prime \prime}$ and $\mathrm{G}^{\prime \prime \prime}$ are the set of normal nodes, advanced nodes and set of super nodes that has not become CHs in the past respectively, so ensuring that the equations (3), (4) and (5) are working.

Average total number of $\mathrm{CHs}$ per round will be:

$$
n .(1-m-b) \cdot p n r m+n \cdot b \cdot p s u p+n \cdot m \cdot p a d v=n \cdot p o p t
$$

Although, average number of $\mathrm{CHs}$ is same as that of DEEC and TSEP, however, here a good aspect of CHRP is that energy dissipation is condensed due to energy heterogeneity and node pairing.

\section{Data Transmission and Data Aggregation}

At the start of each round, the phenomenon of cluster change takes place. In case of CHRP, at cluster change time, the $\mathrm{CH}$ broadcasts the following parameters.

Hard Threshold (HT): It is an absolute value of sensed attribute beyond which node will pass on data to $\mathrm{CH}$. As if sensed value becomes equal to or greater than this threshold value, node turns on its transmitter and transmits.

Soft Threshold (ST): It is the smallest sensed value at which the nodes switch on their transmitters and sends that information to $\mathrm{CH}$.

All nodes keep on sensing the environment continuously. When parameters from attribute set reaches hard threshold value, transmitter is switched on and data is transmitted to $\mathrm{CH}$, however this is only for the first time when this condition is met.

This sensed value is stored in an internal variable in the node, called Sensed Value (SV). Then for the second time and the other, nodes will transmit data if and only if sensed value is greater than hard threshold value or if the difference between currently sensed value and the value stored in SV variable is equal to or greater than the soft threshold [12, 20]. So, by keeping these both thresholds in consideration, the number of data transmissions can be decreased, as transmission will only take place when sensed value reaches hard threshold. And further transmissions are narrowed by soft threshold, as it will eliminate transmissions when there is a small change in value, even smaller than interest. Some of the important features are described below:

1) Time with which critical data reaches the user almost instantaneously.

2) Nodes keep on sensing continuously, but transmission is not done frequently, so energy consumption is much lesser than that of proactive networks.

3) At time of cluster change, values of soft threshold, TR is transmitted afresh and so, user can decide how often to sense and what parameters to be sensed according to criticality of sensed attribute and application.

4) The user can change the attributes depending on requirement, as the attributes are broadcasted at the cluster change time.

The significant trade off of this scheme is that if threshold is not reached, user will not get any information from network and even if any or all the nodes die, system will not 
come to know about that. So, it is not useful for those types of applications where a data is required continuously.

\section{Algorithm Process}

Step 1: For a given simulation time $T$, the Base Station broadcasts HELLO packets periodically. If a sensor node listens HELLO packet then it replies with residual energy. If the RSSI of the received signal is greater than that of any other cluster head then no need to form clusters. Else Clustering occurs.

Step 2: If any node is alive then node pairing is done with closest neighbor. Follow step 4 to decide about the active nodes. If node is active then for each round the Base Station chooses CHs. If a node is Cluster Head then it broadcasts its advertisement message and all non-CHs active nodes, send joining request message to that $\mathrm{CH}$, from which it received the highest RSSI [6, 17]. Cluster head accepts the joining request. Thus forming respective clusters.

Step 3: A node broadcasts a request message to find the closest neighbor. The neighbors respond reliably. The neighbor with the strongest RSSI is selected as the next node. The node status will be PAIRED and it broadcasts Node_Paired_ID message.

Step 4: Get N paired node sets in the network. If node is paired then for each pair set, node broadcasts an Energy_Msg message to its neighboring node $[1,14,16]$. Receiving Energy_Msg from its neighboring node, the node updates its own Neighbor_Table. If $E(r)_{\text {node }}>E(r)_{\text {neighbor }}$ then node is active else the node sleeps. If neighbor node is dead then another node of that pair is awake for remaining rounds else node is active for whole network life time.

Step 5: Base Station receives the status of the residual energy from all nodes. Then based on this, the Base Station computes average energy of the active nodes. For each node having residual energy more than average energy include in the set of eligible Cluster Heads. If a node finds its residual energy more than average energy then it sends a request message to find eligible neighbors. The 1-hop neighbor nodes closer to that node send a reply with their energy consumption rate [21]. The node which has minimum energy consumption rate and with residual energy more than average energy is selected as Cluster Head node.

Step 6: If current value is greater than or equal to the hard threshold then test $=$ current value - sensed value [8]. If test is greater than or equal to the sensed value and the distance between the sensed region is more than threshold then:

Energy $=$ Energy - less transmission energy

else

Energy $=$ Energy - more transmission energy

\section{Simulation Results}

For performance evaluation MATLAB is used. The goals in doing simulations were to compare performance of CHRP with TSEP [6] and DEEC [1] protocols on the basis of energy dissipation with longevity of the network $[5,13]$.
Performance attributes used in the simulations are:

1) Stability period, the period from starting of the network operation and the first dead node.

2) Instability period, the period between the first dead node and the last dead node.

3) Number of nodes alive per round.

4) Number of nodes dead per round.

5) Throughput, number of packets sent from the cluster heads to the base station per unit time.

A network consisting of 100 nodes, placed randomly in a square region, $100 \mathrm{~m} \times 100 \mathrm{~m}$ and a BS located in the center is considered. Simulations for different values of $\alpha$ and $\mathrm{m}$ while keeping $\mathrm{b}$ constant i.e., 0.3 are performed. For the first case $\alpha=1, m=0.2$, for second case $\alpha=3$ and $m=0.2$. This is done to observe the change in network's stability, life and throughput relative to increase the number of advance nodes and corresponding energies. Since $p_{\text {opt }}=0.1$, is optimal probability of CHs, by using equations (3), (4) and (5) different probabilities for every type of node in accordance with different values of $\alpha$ and $m$ are obtained. All the parameters used in simulations are shown in Table I.

TABLE I.

PARAMETER SETTINGS

\begin{tabular}{|c|c|}
\hline Parameters & Value \\
\hline $\mathrm{E}_{\text {elect }}$ & $50 \mathrm{~nJ} / \mathrm{bit}$ \\
\hline $\mathrm{E}_{\mathrm{DA}}$ & $5 \mathrm{~nJ} / \mathrm{bit} / \mathrm{message}$ \\
\hline$\varepsilon_{\mathrm{fs}}$ & $10 \mathrm{pJ} / \mathrm{bit} / \mathrm{m}^{2}$ \\
\hline$\varepsilon_{\mathrm{mp}}$ & $0.0013 \mathrm{pJ} / \mathrm{bit} / \mathrm{m} 4$ \\
\hline $\mathrm{E}_{\mathrm{o}}$ & $0.5 \mathrm{~J}$ \\
\hline$K$ & 4000 \\
\hline $\mathrm{p}_{\mathrm{opt}}$ & 0.1 \\
\hline$N$ & 100 \\
\hline$\alpha$ & 1 \\
\hline$m$ & 0.2 \\
\hline
\end{tabular}

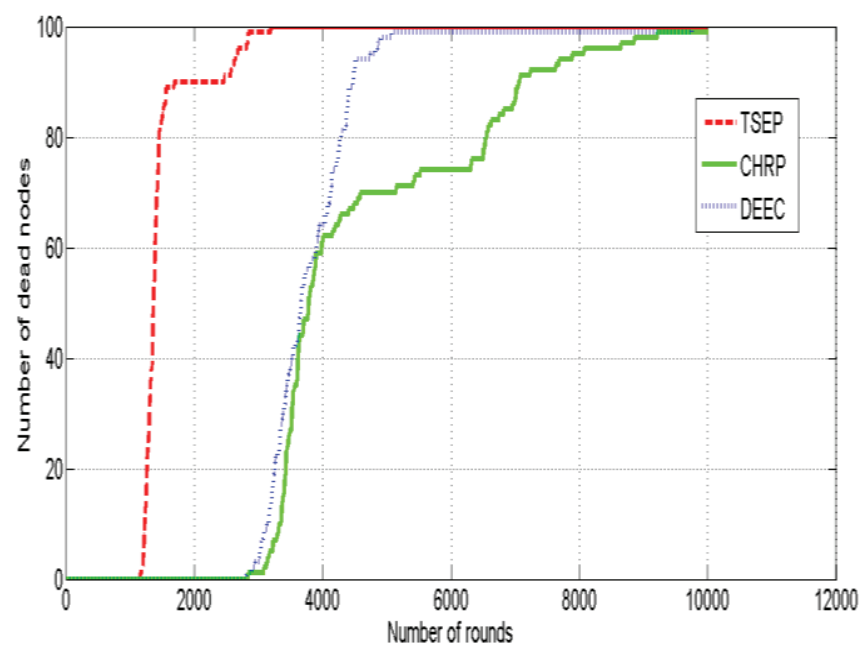

Figure 2. Number of dead nodes per round for $\alpha=1$ and $m=0.2$

By using the equations (6), (7) and (8), CHs election for normal, advance and super nodes respectively, can be determined. 


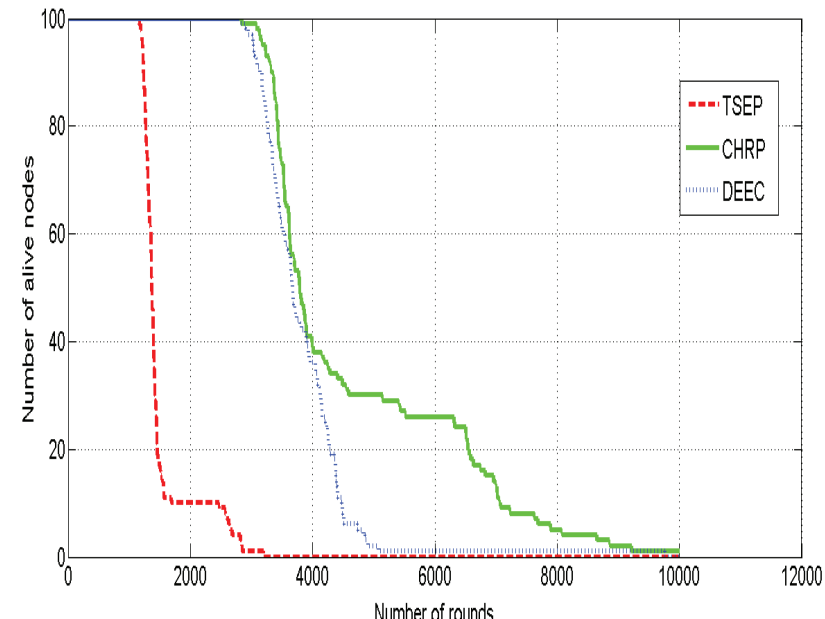

Figure 3. Number of alive nodes per round for $\alpha=1$ and $m=0.2$

Fig.2 and Fig.3 show comparison of protocols DEEC, TSEP and CHRP regarding dead and alive nodes, relative to the number of rounds. As it can be seen, CHRP has completely dominated the other two protocols because of heterogeneity, threshold consideration and node pairing.

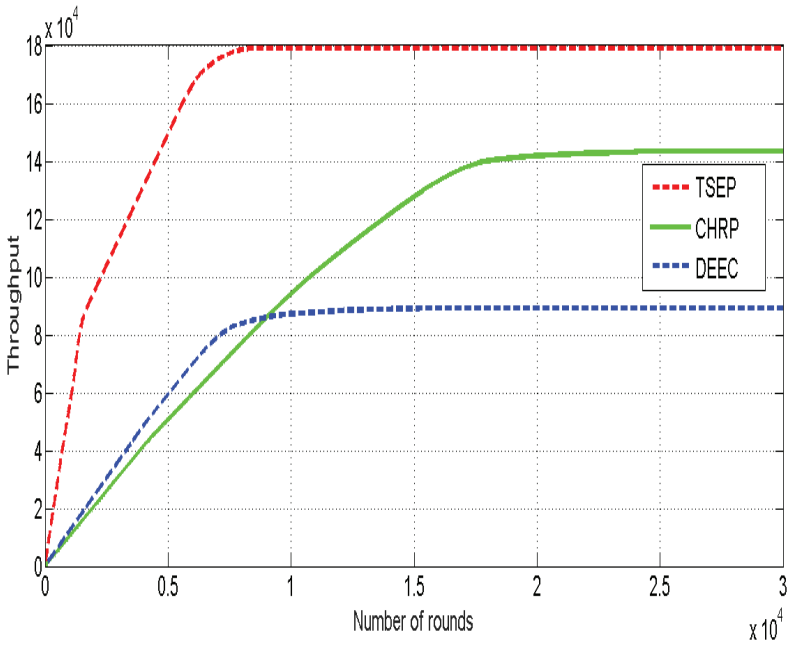

Figure 4. Number of packets sent from $\mathrm{CHs}$ to BS for $\alpha=1$ and $m=0.2$

Fig. 4 shows throughput, data sent from $\mathrm{CHs}$ to the BS. CHRP being threshold sensitive protocol and sleeping protocol, shows better result than DEEC and TSEP, as here transmission rate is less so energy consumption will be less than others.

From Fig. 2, Fig. 3 and Fig. 4, it can be concluded that stability period and the network life time are greater in CHRP, than all other protocols. Nodes tend to die out slowly in CHRP, as in CHRP a huge part of energy is consumed in sensing; while transmission of data is done only at the conditions when HT value is achieved by sensed node or is exceeded.

The number of rounds is more in CHRP when percentage of dead nodes is $50 \%$ and the number of rounds becomes huge in CHRP when percentage of dead nodes is $100 \%$. So, CHRP is a lot better than the existing techniques when $\alpha=1$ and $m=0.1$ as shown in Table II.
TABLE II.

AVERAGE LIFETIME OF NODES IN NUMBER OF NODES FOR $\alpha=1$ AND $m=0.1$

\begin{tabular}{|c|c|c|c|}
\hline \multirow{2}{*}{ \% of dead nodes } & \multicolumn{3}{|c|}{ Number of rounds } \\
\cline { 2 - 4 } & DEEC & TSEP & CHRP \\
\hline 1 & 1020 & 2187 & 3104 \\
\hline 10 & 1038 & 3124 & 3154 \\
\hline 50 & 1201 & 3297 & 3472 \\
\hline 100 & 3211 & 7601 & 9304 \\
\hline
\end{tabular}

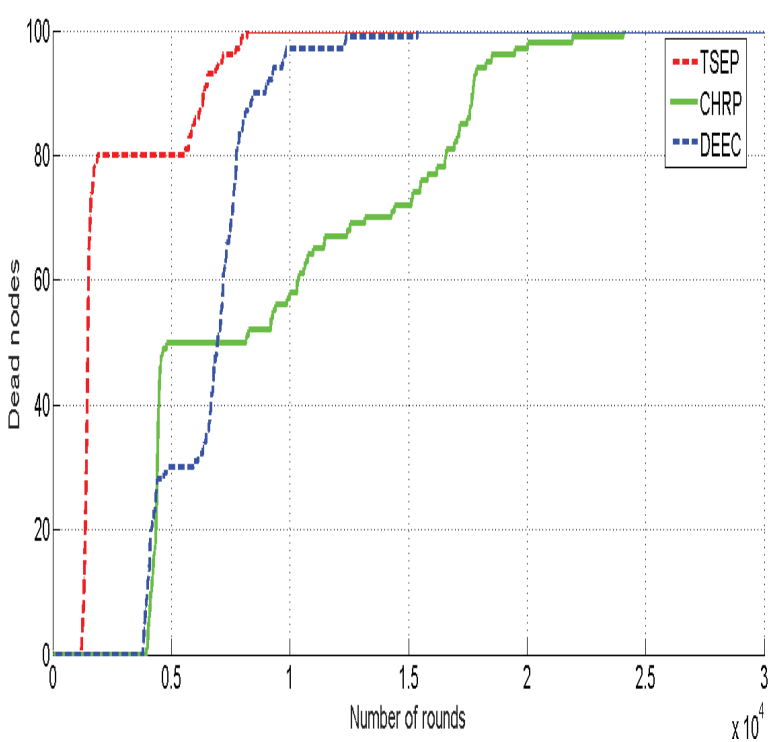

Figure 5. Number of dead nodes per round for $\alpha=2$ and $m=0.3$

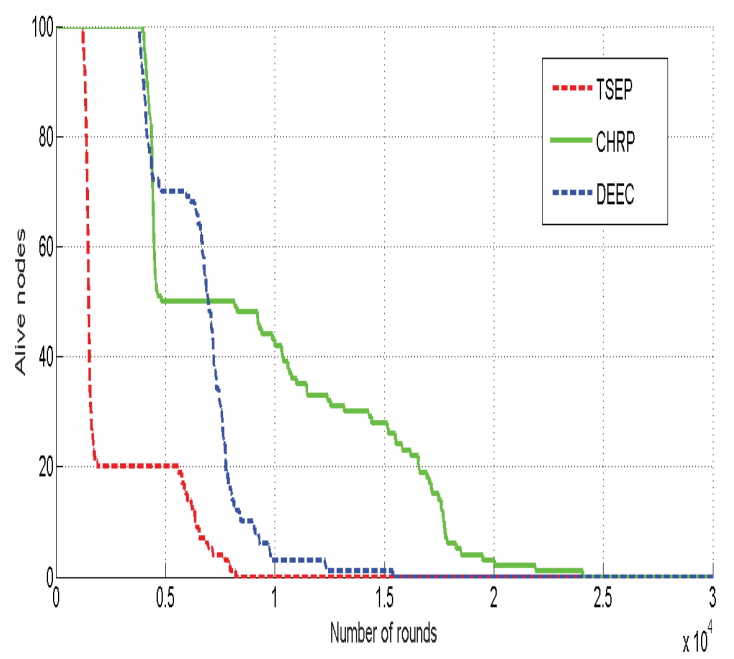

Figure 6. Number of alive nodes per round for $\alpha=2$ and $m=0.3$

Same is the case for results shown in Fig. 5, and Fig. 6, where $\alpha=2$ and $m=0.3$. In this case, energy of nodes as well as packets to BS is also increased. Therefore, more number of nodes will be available with an extra energy. As shown in Fig. 5, Fig. 6, by increasing number of advanced nodes and $\alpha$ stability period and the network lifetime are increased. It happens because of three level heterogeneity. So, it can be clearly seen that there are noticeable differences among the protocols in accordance with alive nodes, dead nodes and throughput. Throughput of DEEC is found to be better but redundant information is reduced in packets sent by CHRP. 
By performing simulations in MATLAB, it is observed that:

- $\quad$ CHRP has enhanced stability period compared to all other protocols. This is shown in Fig. 2, Fig. 3 and Fig. 5, Fig. 6.

- The network life time for CHRP was increased as compared to others.

- Decrease and increase in number of dead and alive nodes respectively with increase in $\alpha$ and $m$.

- Throughput is increased due to three level heterogeneity and there is decrease in throughput due to threshold sensitivity as can be observed in Fig.4.

In Table III, the number of rounds is more in TSEP than that of CHRP when percentage of dead nodes is $50 \%$ but the number of rounds becomes huge in CHRP when \% of dead nodes is $100 \%$. So, CHRP is a lot better than the existing techniques when $\alpha=2$ and $m=0.3$.

TABLE III.

AVERAGE LIFETIME OF NODES IN NUMBER OF NODES FOR $\boldsymbol{a}=2$ AND $m=0.3$

\begin{tabular}{|c|c|c|c|}
\hline \multirow{2}{*}{$\begin{array}{c}\text { \% of dead } \\
\text { nodes }\end{array}$} & \multicolumn{3}{|c|}{ Number of rounds } \\
\cline { 2 - 4 } & DEEC & TSEP & CHRP \\
\hline 1 & 1074 & 4122 & 4127 \\
\hline 10 & 1098 & 4159 & 4182 \\
\hline 50 & 1192 & 6421 & 4306 \\
\hline 100 & 7967 & 15620 & 25098 \\
\hline
\end{tabular}

\section{CONCLUSIONS}

A reactive cluster head routing protocol viz. CHRP is proposed in this paper which is composed with three different levels of energies and node pairing. From the simulations results it is concluded that the proposed routing protocol is more energy efficient and hence there is enhancement in the sensor network lifetime, there are more alive nodes in the network if the results of TSEP and DEEC to the proposed protocol are compared. In the existing system transmission of data depends on current energy of nodes and distance between the nodes whereas TSEP algorithm works only on three different levels of energies. The sleeping algorithm improves lifetime of the network. Selection of $\mathrm{CHs}$ is based on threshold value which depends on three levels of heterogeneity and being a reactive routing network protocol causes increase in stability period and network life. As it can be seen from simulation results the throughput has increased so much. In comparison with DEEC and TSEP it is concluded that the protocol will perform well for long time. In the future, intermediate nodes can be introduced in this protocol for increasing the levels of heterogeneity, results in further increase the network lifetime.

\section{REFERENCES}

[1] "An application-specific protocol architecture for wireless microsensornetworks," IEEE Transactions on Wireless Communications, vol.1, no. 4, pp. 660670, October 2002.

[2] W. R. Heinzelman, A. P. Chandrakasan, and H. Balakrishnan "An application-specific protocol architecture for wireless microsensor networks," IEEE Transactions on Wireless Communications, vol. 1, no.4, pp. 660-670, October 2002.

[3] F. Akyildiz, W. Su, Y. Sankarasubramaniam, E. Cayirici, A survey on sensor networks, IEEE communications magazine 40 (8) (2002) 102-114.

[4] Sarkar, A., \& Murugan, T. S. (2019). Cluster head selection for energy efficient and delay-less routing in wireless sensor network. Wireless Networks, 25(1), 303-320.

[5] B. Meenakshi, P. Anandhakumar, "Lifetime extension of wirelesssensor network by selecting two cluster heads and hierarchical routing",IEEE International Conference on
Advances in Computing, Communications and Informatics, 2012.

[6] Babar Nazir, Halabi Hasbullah, "Energy Balanced Clustering in Wireless Sensor Network", IEEE International Conference on Information Technology, 2010.

[7] Sharma, G., \& Kumar, A. "Improved range-free localization for three-dimensional wireless sensor networks using genetic algorithm" Computers \& Electrical Engineering. Vol. 72, no. 11, pp. 808-827, Nov. 2018.

[8] W. Heinzelman A. Chandrakasan and H. Balakrishnan. An application-specific protocol architecture for wireless microsensor networks. Proc. 33rd Hawaii Int',1. Conf. Sys. Sci., pages 660--670,2000.

[9] Singh, M., Soni, S., \& Kumar, V. G. Sharma, (2016, March). Clustering using fuzzy logic in wireless sensor networks. In 2016 3rd International Conference on Computing for Sustainable Global Development (INDIACom) (pp. 16691674). IEEE.

[10] G. Smaragdakis I. Matta and A. Bestavros. SEP: A stable election protocol for clustered heterogeneous wireless sensor networks. In Second International Workshop on Sensor and Actor Network Protocols and Applications (SANPA), 2004.

[11] Q. Nadeem M.B. Rasheed N. Javaid Z.A. Khan Y. Maqsood and A. Din. M-GEAR: Gateway-based energy-aware multihop routing protocol for wsns. Broadband and Wireless Computing,Communication and Applications (BWCCA) of IEEE, pages 164--169,July 2013.

[12] G. Sharma and A. Kumar, "Modified Energy-Efficient RangeFree Localization Using Teaching-Learning-Based Optimization for Wireless Sensor Networks," IETE Journal of Research, vol. 64, no. 1, pp. 124-138, Jul. 2017.

[13] Sharma, G., \& Kharub, M. (2019). Enhanced Range Free Localization in Wireless Sensor Networks. CVR Journal of Science and Technology, 16(1), 26-31.

[14] G. Sharma, \& A. Kumar, "Improved DV-Hop localization algorithm using teaching learning based optimization for wireless sensor networks". Telecommunication Systems, vol. 67, no. 2, pp. 163-178, 2017.

[15] R. Mitra and D. Nandy. A survey on clustering techniques for wireless sensor network. Int. J. of Research in Computer Science, 2(4):51--57,2012.

[16] Prasad, A. Y., \& Balakrishna, R. (2019). Implementation of optimal solution for network lifetime and energy consumption metrics using improved energy efficient LEACH protocol in MANET. Telkomnika, 17(4), 1758-1766

[17] G. Sharma and A. Kumar, "Fuzzy logic based 3D localization in wireless sensor networks using invasive weed and bacterial foraging optimization," Telecommunication Systems, vol. 67, no. 2, pp. 149-162, May 2017.

[18] A. Kashaf, N. Javaid, Z. A. Khan and I. A. Khan. TSEP: Threshold-sensitive Stable Election Protocol for WSNs, Cornell University Library, 2012.

[19] W. R. Heinzelman, A. P. Chandrakasan, and H. Balakrishnan, "Energy Efficient communication protocol for wireless microsensor networks," in Proceedings of the 33rd Hawaii International Conference on System Sciences (HICSS-33), January 2000.

[20] G. Sharma, \& A. Kumar, "Dynamic Range Normal Bisector Localization Algorithm for Wireless Sensor Networks". Wireless Personal Communications, vol. 9, no. 3, pp. 45294549, 2017.

[21] Wang, Q., Lin, D., Yang, P., \& Zhang, Z. (2019). An EnergyEfficient Compressive Sensing-Based Clustering Routing Protocol for WSNs. IEEE Sensors Journal, 19(10), 39503960. 